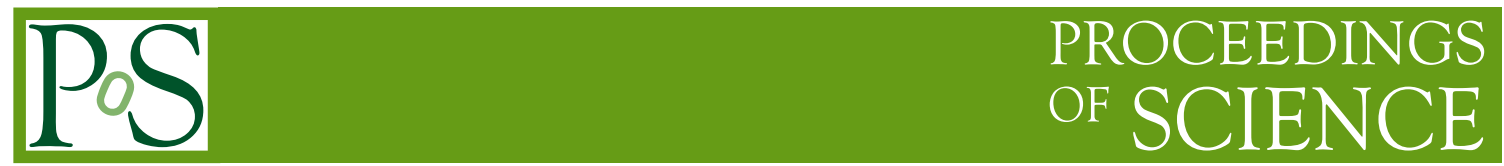

\title{
Charm status and prospects at Belle II
}

\author{
Jitendra Kumar ${ }^{1, *}$ \\ Department of Physics, \\ Carnegie Mellon University, \\ 5000 Forbes Ave, Pittsburgh, US
}

E-mail: jkumar@andrew.cmu.edu

The Belle II experiment started collecting data in summer 2018 and since then more than $176 \mathrm{fb}^{-1}$ of integrated luminosity has been collected. With the current sample size, charm measurements are focused mostly on early reconstruction and studies of detector performance. In this proceeding, we present the current status and prospects of the charm measurements with the target luminosity of $50 \mathrm{ab}^{-1}$ at Belle II. In particular, the $D^{0}$ and $D^{+}$lifetime study, time-integrated charge-parity $(C P)$ asymmetries, $D^{0}-\bar{D}^{0}$ mixing and decay-time-dependent $C P$ violation measurements are discussed.

\footnotetext{
*** 10th International Workshop on Charm Physics (CHARM2020), ***

***31 May - 4 June, 2021 ***

*** Mexico City, Mexico - Online ***
}

${ }^{1}$ On behalf of the Belle II collaboration

${ }^{*}$ Speaker 


\section{Introduction}

The charm sector offers potential to search for physics beyond the Standard Model (SM). Charm is the only up-type quark to exhibit mixing, however this mixing is heavily suppressed. Charge-parity violation (CPV) in charm is also suppressed. Belle [1] and BaBar [2] reported the first evidence of charm mixing in 2007 but CPV observation took an additional decade until LHCb achieved this milestone in 2019 [3]. The Belle II experiment provides excellent opportunities to perform charm measurements as large samples of $B$ and $D$ decays $\left(6.5 \times 10^{10}\right.$ pairs of $b$ and $\left.c\right)$ will be collected with the planned integrated luminosity of $50 \mathrm{ab}^{-1}$. The LHCb at the CERN will collect even more, but Belle II has other advantages such as the lower backgrounds from $e^{+} e^{-}$collisions, excellent reconstruction of neutral particles $\left(\pi^{0}, \gamma\right.$ and $\left.\eta\right)$ and, acceptance and trigger criteria that introduce much less bias on lifetime and kinematic properties. The missing energy and missing mass based analysis [9] are also straightforward as the initial state energy and momentum are known in Belle II. The core of Belle II charm physics program will be focused on charm decays with $\pi^{0}$ in their final states.

\section{SuperKEKB and Belle II Detector}

SuperKEKB, located at KEK, Tsukuba, Japan, collides $e^{+}$and $e^{-}$with asymmetric energies at centre-of-mass energy of $10.58 \mathrm{GeV}$, the mass of $\Upsilon(4 S)$ resonance. This second generation $B$ factory is expected to deliver about $\sim 30$ times higher instantaneous luminosity $\left(6.5 \times 10^{35} \mathrm{~cm}^{-2} \mathrm{~s}^{-1}\right)$ as compared to its previous generation KEKB, mostly due to reduction in the the beam size (nanobeam scheme) by a factor 20 and an increase in currents by factor 2 . In June 2021, SuperKEKB achieved the world record instantaneous luminosity of $3.1 \times 10^{34} \mathrm{~cm}^{-2} \mathrm{~s}^{-1}$.

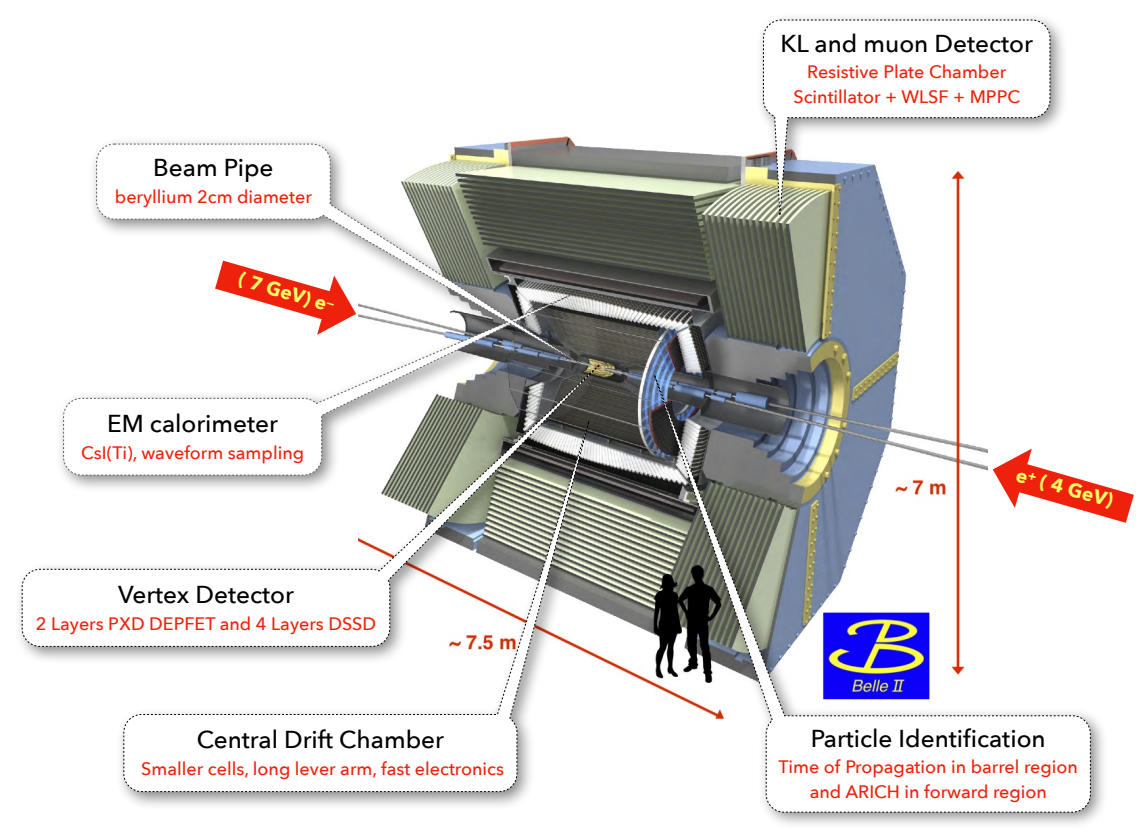

Figure 1: Schematic of the Belle II detector. 
The Belle II detector, shown in Figure 1, is the next-generation successor to the Belle detector, equipped with major upgrades to deal with higher background levels and pile-up expected with the high luminosity environment of SuperKEKB. The interaction point of Belle II is surrounded by a beam pipe with a diameter of $2 \mathrm{~cm}$. Around the beam pipe are a vertex silicon-pixel detector for precise decay vertex reconstruction and four layers of double-sided silicon vertex detector (SVD) for tracking and vertex reconstruction. The SVD is also used in particle identification (PID) based on specific ionization energy loss $(d \mathrm{E} / d x)$. The central drift chamber has 56 measurement-layers immersed in a 50:50 mixture of helium and ethane. It is the main tracking detector and provides PID via $d \mathrm{E} / d x$. The time-of-propagation (TOP) counter, based on a Cherenkov device in the central barrel region, is the main PID detector and provides excellent $K-\pi$ separation. The TOP PID is complemented by the ARICH, a aerogel-based proximity focusing ring-imaging Cherenkov system in the forward end-cap region. The electromagnetic calorimeter, based on Belle's CsI(Tl) crystal calorimeter, but with new front-end electronics and waveform fitting algorithm, is used for electron and photon reconstruction. Finally, an improved $K_{L}$ and $\mu$ detector, composed of layers of iron sandwiched with resistive plate chamber or plastic scintillator, is used for identification of $\mu$ and $K_{L}$. In addition to the detector upgrades, the trigger, data acquisition system and computing are also upgraded to deal with the higher event rate in Belle II.

\section{Charm measurements at Belle II}

A large part of the Belle II charm physics program aims at high-precision measurements of time-integrated CPV, charm mixing and mixing-induced CPV. The major improvement comes from the new vertex detector; it provides $D^{0}$ decay time resolution twice as better than Belle and BaBar [7]. This resolution improvement will benefit time-dependent analyses of charm mixing and indirect CPV. The other upgrades are also expected to improve the overall performance, yielding improved tracking efficiency, good PID even with higher beam background, and improved $K_{S}$ efficiency. As of June 4, 2021, the Belle II experiment collected $176 \mathrm{fb}^{-1}$ of data and current charm measurements with a subset of these data are focused on performance studies.

\section{1 $D^{0}$ and $D^{+}$lifetime measurements}

Belle II reports the most precise measurements of the $D^{0}$ and $D^{+}$lifetimes using $D^{*+} \rightarrow D^{0}(\rightarrow$ $\left.K^{-} \pi^{+}\right) \pi^{+}$and $D^{*+} \rightarrow D^{+}\left(\rightarrow K^{-} \pi^{+} \pi^{+}\right) \pi^{0}$ decays [4]. The $D^{0}$ and $D^{+}$reconstruction and selection of signal candidates are first optimzed with simulations and then analyses are performed on a data set with integrated luminosity of $72 \mathrm{fb}^{-1}$. In both analyses, a vertex fit [5] is performed on the global decay-chain, which constrains the tracks according to the decay topology and the $D^{*+}$ candidate to originate from the interaction region. A $\pi^{0}$-mass constraint is additionally applied in the $D^{+}$ decay-chain fit. The charmed mesons originating from decays of bottom mesons are suppressed by requiring the $D^{*+}$ momentum threshold of $2.5(2.6) \mathrm{GeV} / c$ in the $D^{0}\left(D^{+}\right)$analysis. Finally, in events with multiple $D^{*+}$ candidates, a randomly selected candidate is retained. Background in the $D^{0}$ signal region is at the per-mille level and about $9 \%$ in the $D^{+}$signal region, they arise from misreconstructed charmed decays and combinatorics from random tracks. The lifetime is then extracted with a likelihood fit to the unbinned distributions of the lifetime and its uncertainty. The background contribution is neglected in $D^{0}$ decay-time fits and a systematic uncertainty is assigned whereas 
the background is modeled using sidebands in $D^{+}$decay-time fits. The decay-time distributions with fit projections are shown in Figure 2. The results, $\tau\left(D^{0}\right)=410.5 \pm 1.1$ (stat) \pm 0.8 (syst) fs and $\tau\left(D^{+}\right)=1030.4 \pm 4.7$ (stat) \pm 3.1 (syst) fs, are the world's most accurate measurements and agree with previous measurements. The systematic uncertainties arise from the sources listed in Table 1.

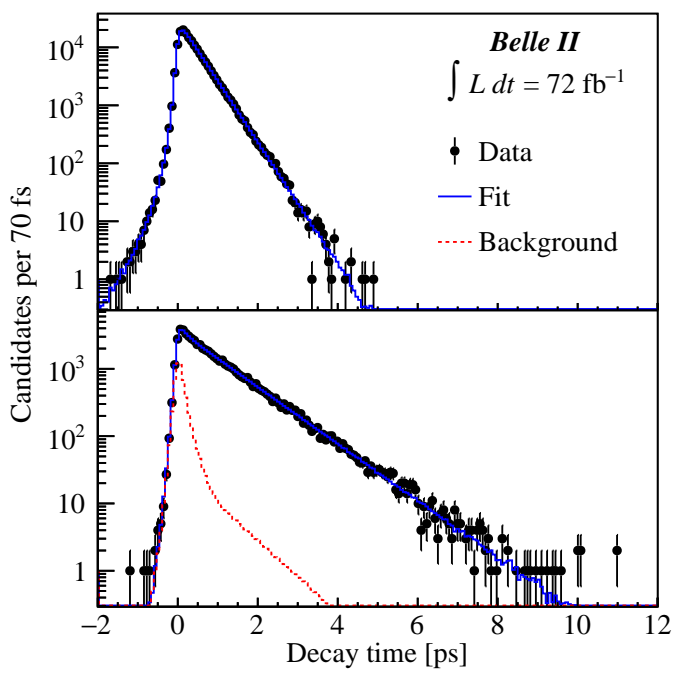

Figure 2: Decay-time distributions of $D^{0} \rightarrow$ $K^{-} \pi^{+}$(top) and $D^{+} \rightarrow K^{-} \pi^{+} \pi^{+}$(bottom) candidates.

\begin{tabular}{lcc}
\hline Source & $\tau\left(D^{0}\right)[\mathrm{fs}]$ & $\tau\left(D^{+}\right)[\mathrm{fs}]$ \\
\hline Resolution model & 0.16 & 0.39 \\
Backgrounds & 0.24 & 2.52 \\
Detector alignment & 0.72 & 1.70 \\
Momentum scale & 0.19 & 0.48 \\
\hline Total & 0.80 & 3.10 \\
\hline
\end{tabular}

Table 1: Systematic uncertainties [4].

\section{2 $D^{0}-\bar{D}^{0}$ mixing and indirect $\mathrm{CPV}$ measurements}

The excellent $D^{0}$ decay-time resolution reported by Belle II [4] will benefit precision measurements of mixing parameters $(x=\Delta \mathrm{M} / \Gamma, y=\Delta \Gamma / 2 \Gamma)^{1}$ and CPV parameters $(|q / p|, \arg (q / p)=\phi)$. A sensitivity estimation based on simulated $D^{0} \rightarrow K^{-} \pi^{+}$[9] decay shows a significant improvement in precision compared to Belle and BaBar.
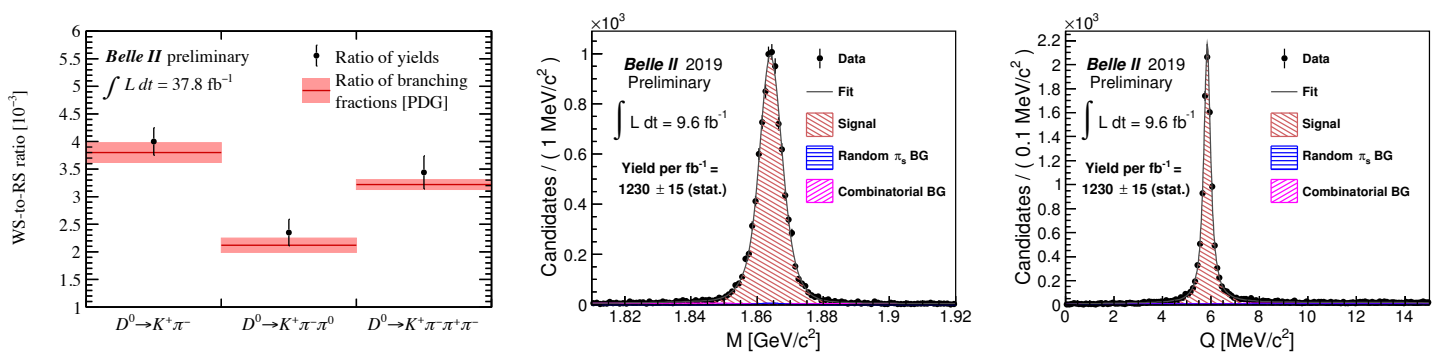

Figure 3: The wrong-sign (WS) to right-sign (RS) ratio for WS $D^{0}$ decays (left) and $D^{0}$ mass $\mathrm{M}$ (middle) and Q resolution (right) distributions for $D^{0} \rightarrow K_{s} \pi^{+} \pi^{-}$decay.

Ongoing Belle II measurements of wrong-sign $D^{0}$ decays and the Dalitz analysis of $D^{0} \rightarrow$ $K_{S} \pi^{+} \pi^{-}$are at preliminary stage due to limited sample size. In the wrong-sign $D^{0}$ decays analysis,

${ }^{1} \Delta \mathrm{M}$ and $\Delta \Gamma$ are mass and decay width difference of the $C P$-even and $C P$-odd eigenstate 
several ratios of wrong-sign (WS) to right-sign (RS) decay yields (with statistical uncertainties only) are measured using an integrated luminosity of $37.8 \mathrm{fb}^{-1}$. The results are illustrated in Figure 3 (left) and are compatible with the world-average ratios. The Dalitz analysis of $D^{0} \rightarrow K_{S} \pi^{+} \pi^{-}$shows good reconstruction performance and a twice better $\mathrm{Q}\left(=M\left(D^{*+}\right)-M\left(D^{0}\right)-m_{\pi^{+}}\right)$resolution as compared to the Belle measurement. The results are obtained with an integrated luminosity of 9.6 $\mathrm{fb}^{-1}$ and illustrated in Figure 3 (middle and right).

\subsection{Time integrated CPV measurements}

The excellent reconstruction efficiency and small detector-induced asymmetries makes Belle II an ideal experiment for searching for time-integrated CPV in charm decays $A_{C P}=\left[\Gamma\left(D^{0}\right)-\right.$ $\left.\Gamma\left(\bar{D}^{0}\right)\right] /\left[\Gamma\left(D^{0}\right)+\Gamma\left(\bar{D}^{0}\right)\right]$. Table 2 shows the $A_{C P}$ measurements for various decays by the Belle experiment and their projections in Belle II with the target luminosity of $50 \mathrm{ab}^{-1}$. In projections, uncertainties are estimated using the equation $\sigma_{\text {Belle II }}=\sqrt{\left(\sigma_{\text {stat }}^{2}+\sigma_{\text {syst }}^{2}\right) \cdot\left(\mathscr{L}_{\text {Belle }} / 50 \mathrm{ab}^{-1}\right)+\sigma_{\text {irred }}^{2}}$.

\begin{tabular}{l|ccc} 
Mode & $\mathscr{L}\left(\mathrm{fb}^{-1}\right)$ & $A_{C P}(\%)$ & Belle II $50 \mathrm{ab}^{-1}$ \\
\hline$D^{0} \rightarrow K^{+} K^{-}$ & 976 & $-0.32 \pm 0.21 \pm 0.09$ & \pm 0.03 \\
$D^{0} \rightarrow \pi^{+} \pi^{-}$ & 976 & $+0.55 \pm 0.36 \pm 0.09$ & \pm 0.05 \\
$D^{0} \rightarrow \pi^{0} \pi^{0}$ & 966 & $-0.03 \pm 0.64 \pm 0.10$ & \pm 0.09 \\
$D^{0} \rightarrow K_{S}^{0} \pi^{0}$ & 966 & $-0.21 \pm 0.16 \pm 0.07$ & \pm 0.02 \\
$D^{0} \rightarrow K_{S}^{0} K_{S}^{0}$ & 921 & $-0.02 \pm 1.53 \pm 0.02 \pm 0.17$ & \pm 0.23 \\
$D^{0} \rightarrow K_{S}^{0} \eta$ & 791 & $+0.54 \pm 0.51 \pm 0.16$ & \pm 0.07 \\
$D^{0} \rightarrow K_{S}^{0} \eta^{\prime}$ & 791 & $+0.98 \pm 0.67 \pm 0.14$ & \pm 0.09 \\
$D^{0} \rightarrow \pi^{+} \pi^{-} \pi^{0}$ & 532 & $+0.43 \pm 1.30$ & \pm 0.13 \\
$D^{0} \rightarrow K^{+} \pi^{-} \pi^{0}$ & 281 & $-0.60 \pm 5.30$ & \pm 0.40 \\
$D^{0} \rightarrow K^{+} \pi^{-} \pi^{+} \pi^{-}$ & 281 & $-1.80 \pm 4.40$ & \pm 0.33 \\
\hline$D^{+} \rightarrow \phi \pi^{+}$ & 955 & $+0.51 \pm 0.28 \pm 0.05$ & \pm 0.04 \\
$D^{+} \rightarrow \pi^{+} \pi^{0}$ & 921 & $+2.31 \pm 1.24 \pm 0.23$ & \pm 0.17 \\
$D^{+} \rightarrow \eta \pi^{+}$ & 791 & $+1.74 \pm 1.13 \pm 0.19$ & \pm 0.14 \\
$D^{+} \rightarrow \eta^{\prime} \pi^{+}$ & 791 & $-0.12 \pm 1.12 \pm 0.17$ & \pm 0.14 \\
$D^{+} \rightarrow K_{S}^{0} \pi^{+}$ & 977 & $-0.36 \pm 0.09 \pm 0.07$ & \pm 0.03 \\
$D^{+} \rightarrow K_{S}^{0} K^{+}$ & 977 & $-0.25 \pm 0.28 \pm 0.14$ & \pm 0.05 \\
\hline$D_{S}^{+} \rightarrow K_{S}^{0} \pi^{+}$ & 673 & $+5.45 \pm 2.50 \pm 0.33$ & \pm 0.29 \\
$D_{S}^{+} \rightarrow K_{S}^{0} K^{+}$ & 673 & $+0.12 \pm 0.36 \pm 0.22$ & \pm 0.05 \\
\hline
\end{tabular}

Table 2: Time-integrated (direct) $C P$ asymmetries measured by Belle, and the precision expected for Belle II with $50 \mathrm{ab}^{-1}$ of data (reproduced from [9]).

The statistical uncertainties $\left(\sigma_{\text {stat }}\right)$ are scaled with the luminosity ratio. The systematic uncertainties $\left(\sigma_{\text {syts }}\right)$ that scale with sample-size, such as background shapes are also scaled with the luminosity ratio whereas the irreducible ones, such as those from decay time resolution due to detector misalignment are unchanged. Figure 4 shows $\Delta M$ distributions, the mass difference between $D^{*+}$ and $D^{0}$, from ongoing charm measurements at Belle II. The results, even with current 
statistics not only show very good reconstruction performance but also their resolution, background and signal yield are comparable or better than in Belle measurements [8].
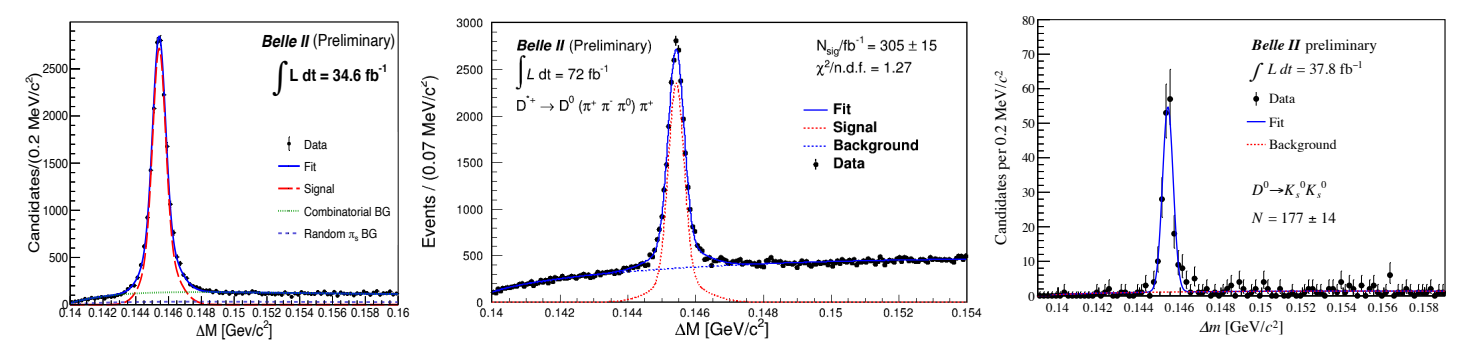

Figure 4: $\Delta M$ distributions for $D^{*+} \rightarrow D^{0}\left(\rightarrow K_{s}^{0} \pi^{0}\right) \pi^{+}$(left), $D^{*+} \rightarrow D^{0}\left(\rightarrow \pi^{+} \pi^{-} \pi^{0}\right) \pi^{+}$(middle) and $D^{*+} \rightarrow D^{0}\left(\rightarrow K_{s}^{0} K_{s}^{0}\right) \pi^{+}$(right) decays.

\section{Summary}

Thanks to the extraordinary efforts of colleagues stationed at Tsukuba, Japan, which ensured smooth operation of Belle II and SuperKEKB during the Covid-19 pandemic, the Belle II experiment recorded more than $176 \mathrm{fb}^{-1}$ of integrated luminosity as of June 4, 2020. The goal is to collect $50 \mathrm{ab}^{-1}$ by 2030 . The recent Belle II results on the lifetime measurement of $D^{0}$ and $D^{+}$are the world's best. The other ongoing charm measurements, even with modest sample size, show good reconstruction performance and are also comparable with previous Belle measurements. This is just the beginning of a broad charm program at Belle II, which yields many opportunities with the larger data set.

\section{References}

[1] B. Aubert et al. (BaBar collaboration), "Evidence for $D^{0}-\bar{D}^{0}$ Mixing”, Phys. Rev. Lett. 98, 211802 (2007).

[2] M. Staric et al. (Belle collaboration), “Evidence for $D^{0}-\bar{D}^{0}$ Mixing”, Phys. Rev. Lett. 98, 211803 (2007).

[3] R. Aaij et al. (LHCb collaboration), “Observation of CP Violation in Charm Decays", Phys. Rev. Lett. 122, 211803 (2019).

[4] F. Abudinen et al. (Belle II collaboration), "Precise measurement of the $D^{0}$ and $D^{+}$lifetimes at Belle II", arXiv:2108.03216 (submitted to PRL).

[5] J.-F. Krohn et al. (Belle-II analysis software group), "Global Decay Chain Vertex Fitting at B-Factories”, Nucl. Instrum. Meth. A976, 164269 (2020).

[6] T. Skwarnicki, "A study of the radiative cascade transitions between the Upsilon-prime and Upsilon resonances", Ph.D. thesis, Institute of Nuclear Physics, Krakow., DESY-F31-86-02 (1986). 
[7] G. Casarosa (for the Belle-II collaboration), "Charm Potential at BelleII" at ICHEP 2020, [https://indico.cern.ch/event/868940/contributions/3815673/].

[8] N. Dash et al. (Belle collaboration), "Search for CP Violation and Measurement of the Branching Fraction in the Decay $D^{0} \rightarrow K_{S}^{0} K_{S}^{0}$ ”, Phys. Rev. Lett. 119, 171801 (2017).

[9] E.Kou et al., “The Belle II Physics Book”, PTEP, 2019, 123 C01 (2019); errata 2020, 029201(E) (2020). 